\title{
CONTROLE DA MALHA DE TORQUE COM ACIONAMENTO DE VELOCIDADE APLICADO A UMA BANCADA EXPERIMENTAL PARA ENSAIO DE MOTORES
}

\author{
Rafael da Silva de Sousa *, Victor de Paula Brandão Aguiar ${ }^{\dagger}$, Wilkley Bezerra \\ Correia*, Jucelino Taleires Filho*, Flávio André Azevedo de Campos*, Darielson A. \\ SOUZA* \\ * Universidade Federal do Ceará \\ Campus do Pici \\ Caixa Postal 6001 Fortaleza, Ceará, Brasil \\ $\dagger$ Universidade Federal Rural do Semi-Árido \\ Av. Francisco Mota, 572, Campus Leste \\ 59625-900, Mossoró, Rio Grande do Norte, Brasil
}

Emails: rafaelgow2@dee.ufc.br, victor@ufersa.edu.br, wilkley@dee.ufc.br, taleires@dee.ufc.br, flavio.andre.campos@alu.ufc.br, darielson@dee.ufc.br

\begin{abstract}
This work presents the implementation of control strategies for torque loop control of a 3- $\phi$ indution motor with speed as control variable. So that it regards the viability to run torque control loop asynchronously to inner speed and current loops. The latter runs in a frequency inverter while the former is a control algorithm (LQG or RST) implemented in a computer. The main purpose is driven to observe torque response to a step reference signal, so performance of the controllers may be highlithed.
\end{abstract}

Keywords - torque control, induction motor, LQG, RST

Resumo - Este trabalho apresenta a implementação de estratégias de controle aplicadas à malha de torque de um motor de indução trifásico tendo a velocidade do motor como variável de controle. Dessa forma, pode-se analisar a viabilidade de se implementar o controle de torque de forma assíncrona em relação ao controle de velocidade e corrente das malhas mais internas. Este é implementado em um inversor de frequência, enquanto aquele é um algoritmo de controle (LQG ou RST) implementado em um computador. O principal propósito do estudo está na observação da resposta de torque a uma referência do tipo degrau, assim o desempenho dos controladores é destacado.

Palavras-chave- controle, torque, motor indução, LQG, RST

\section{Introdução}

Em aplicações industriais há necessidade de se avaliar o desempenho de motores elétricos em diferentes tipos de aplicações nas quais o torque e velocidade podem mudar através de uma ampla faixa de valores (Gan et al., 2015; de Oliveira et al., 2018). Atualmente, índices mínimos de rendimento são encorajados em motores sob carga variável (torque e velocidade) e alimentados por inversores visando melhor eficiência energética em sistemas motrizes (Gynther et al., 2016; Waide and Brunner, 2011).

A emulação de sistemas mecânicos é realizada para observação do desempenho de motores sob condições de operação reais. Nesse contexto, o uso de técnicas de acionamento constitui prática comum, desde o controle vetorial de velocidade, o controle direto de torque e o controle de posição (Arellano-Padilla et al., 2006; Gan et al., 2015; de Oliveira et al., 2018). Entretanto, o projeto, controle e operação um sistema motriz, pode ser realizado em laboratório, utilizando-se uma bancada para ensaios de motores. Dessa forma, a medição de rendimento nas máquinas pode ser obtida a partir da emulação de cargas mecânicas reais, em ambientes controlados, semelhante às apresentadas por Quintino et al. (2011) e Agamloh et al.
(2011). Assim, com o propósito de emular um perfil de carga desejado, se faz necessário garantir que o torque desenvolvido em bancada seja também controlado.

Trabalhos recentes evidenciando estratégias diversas de controle de torque, dentre elas o $D i$ rect Torque Control (DTC), mostram avanços nas estratégias de utilizando controladores preditivos junto ao DTC com Filtro de Kalman atuando como observador de fluxo obtendo resposta dinâmica rápida e redução das harmônicas de ondulação de torque e corrente do estator (Ouhrouche et al., 2016).

Neste trabalho aplica-se o controle de torque constante com atuação na malha de velocidade, ou seja, a saída do controlador de torque produz as referências da malha controlada de velocidade. Trata-se de um controle em cascata, sendo a malha de corrente a mais interna, enquanto a malha da variável mecânica (velocidade) posicionase mais externamente. No caso deste trabalho, a malha de torque é ainda mais externa do que a malha de velocidade. Para efeito de projeto do controlador, faz-se necessário identificar a planta, na qual relaciona o set-point de velocidade do motor (entrada) e o torque (saída).

A principal razão para a aplicação do controle de torque constante é que este permite avaliar me- 
lhor a implementação de dois tipos diferentes de controle: o PID, que é um controle tipicamente no domínio da frequência; e um controle LQG, que é tipicamente no domínio do tempo, utilizando espaço de estados. Assim, aspectos relacionados ao sobressinal ou tempo de acomodação ficam mais evidentes na referência ao degrau do que rampa ou parábola.

Além disso, a estratégia de controle implementada neste trabalho considera que as malhas de corrente e velocidade são implementadas diretamente embarcadas no inversor de frequência, enquanto a malha de torque é embarcada em um sistema de controle externo em micro-computador, com interface através de uma placa de aquisição de dados. Assim, a malha mais externa atua de forma assíncrona às internas. E os sinais de controle da malha externa são as referências das malhas internas.

Este artigo apresenta na seção 2, sucintamente, a descrição da bancada utilizada nos testes práticos. Na seção 3, são discutidos detalhes do sistema de acionamento e controle. Na seção 4, são apresentados os resultados dos controladores projetados. As considerações finais e conclusões são apresentadas na $5^{a}$ seção.

\section{Bancada para medição de rendimento de motores}

A bancada é constituída em essência de dois motores de indução trifásicos, o motor sob ensaio (MSE) a um lado do eixo passante e a chamada máquina emuladora (ME) do outro lado. No eixo, tem-se o transdutor de torque (TT), para medição de torque, velocidade e potência. A ME é alimentada pelo inversor que opera em quatro quadrantes (INV4Q) e permite a operação da máquina em modo gerador possibilitando que o conjunto MEINV4Q emule carga mecânica no eixo do MSE. O INV4Q conecta a ME à rede elétrica do laboratório regenerando a energia elétrica durante o ensaio (Agamloh et al., 2011; Quintino et al., 2011).

Este equipamento conta com algoritmo de identificação do tipo self-tuning em que a malha interna de correntes $i_{d}$ e $i_{q}$ é controlada através de controle vetorial com estimação de fluxo e leitura de tensão, enquanto o controlador de velocidade é do tipo PI. A Figura 1 ilustra de forma simplificada a estratégia de controle no inversor em que $G_{e}(s)$ é a função de transferência associada à corrente e $G_{m}(s)$ está relacionada à velocidade.

Há ainda uma placa de aquisição de dados de fabricação da National Instruments ${ }^{\circledR}$, modelo USB-6009, para aquisição de dados no PC, via USB de forma assíncrona. Assim, o fluxo de dados entre a placa e o inversor se dá através de entradas e saídas analógicas. Um algoritmo foi desenvolvido para a identificação da bancada e implementado numa plataforma de aquisição de

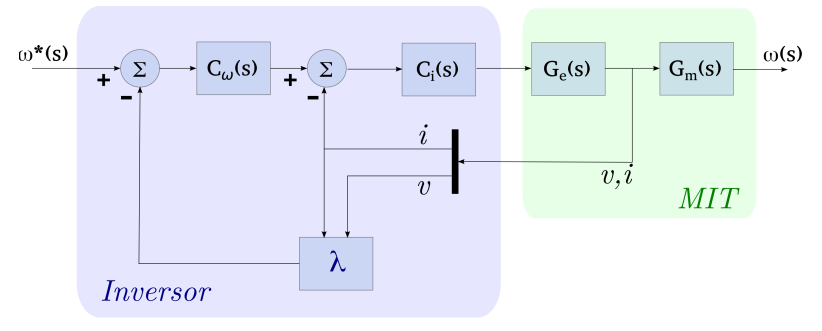

Figura 1: Malhas de controle no inversor

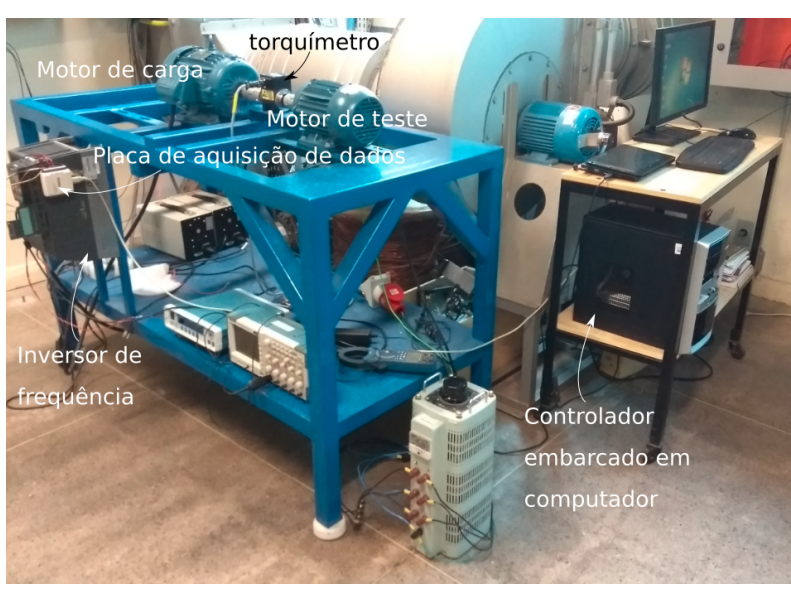

Figura 2: Foto da bancada em estudo.

dados. A identificação engloba a faixa de operação segura entre os dois MIT's e a malha de controle de velocidade do INV4Q. A partir da planta identificada, projeta-se um controlador para uma malha externa de torque. A Figura 2 ilustra a foto da bancada em estudo com os elementos descritos nesta seção.

\section{Acionamento e controle}

Nesta seção descreve-se a forma como a malha externa de torque é implementada em relação ao inversor de frequência, além dos controladores considerados neste estudo.

\subsection{Acionamento do inversor}

O inversor de frequência descrito na seção 2 possui um módulo analógico de entrada e saída de dados em tensão. Dessa forma, é possível efetuar a leitura de variáveis medidas ou estimadas, além de enviar sinais de comando provenientes de fonte externa ao inversor.

O objetivo deste trabalho, como já mencionado na seção 1, consiste no controle de variáveis mecânicas de torque e velocidade, considerando que uma delas já é controlada em cascata com a malha interna de corrente. Assim, neste trabalho as malhas de corrente e velocidade são controladas diretamente no inversor industrial, enquanto a malha de torque é posicionada externamente à malha de velocidade, ou seja, o controlador de tor- 


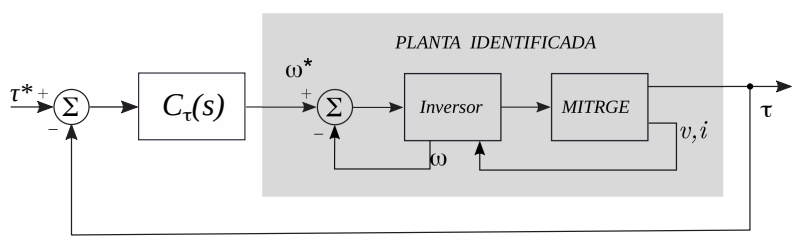

Figura 3: Sistema de controle

que calcula a referência de velocidade. A Figura 3 apresenta o diagrama de blocos do sistema de controle.

O controle de torque, ou seja, a malha mais externa do sistema de controle, deve, portanto, ser realizada em uma unidade de processamento independente, como um controlador lógico programável (CLP), embarcado em placa de desenvolvimento ou em microcomputador. Qualquer que seja uma dessas escolhas, o sinal de comando (referência de velocidade) é enviado de forma assíncrona, bem como a leitura de torque. No entanto, a malha de torque é lenta se comparada com as malhas de variáveis elétricas (corrente e tensão). Dessa forma, no caso deste estudo, a malha mais externa pode operar em modo assíncrono já que o sinal de controle (velocidade) também pertence à malha mecânica.

O controle de torque é realizado em microcomputador, cujos algoritmos são implementados em pacotes computacionais de matemática. A interface de entrada e saída de dados é realizada através de uma placa de aquisição de dados de baixo custo, de fabricação da National Instruments, modelo NI-USB 6009, para a qual já existem bibliotecas disponíveis tanto para Scilab quanto para MATLAB. A Figura 4 apresenta o diagrama esquemático de acionamento da bancada

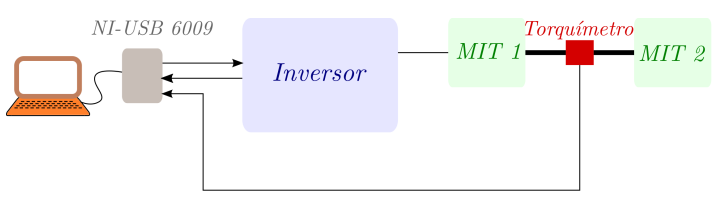

Figura 4: Diagrama esquemático de acionamento da bancada

\subsection{Estratégias de controle}

Duas estratégias de controle são consideradas neste artigo: um controlador PI escrito na forma RST e uma variação do controlador LQG com ajuste dado por filtro de robustez. A ideia não é de comparar os resultados dos dois controladores, mas de apresentar a viabilidade de implementação de um controlador bastante popular e conhecido, como é o caso do PI, e de um controlador em espaço de estados. Nesse contexto, uma vez que se deseja avaliar o desempenho de controladores, torna-se adequado considerar o seguimento de referência do tipo degrau.

\subsection{Identificação da planta}

O sistema de controle que se deseja controlar é composto pela malha fechada de velocidade, incluindo o controlador de velocidade e de corrente, apresentado na Figura 3. Dessa forma, tornase convenente identificar esse sistema como uma planta única, a partir da aplicação de um sinal PRBS-analógico na estrada de referência de velocidade. Esse sinal de excitação (PRBS-analógico) consiste em uma sequência de degraus que podem assumir um valor aleatório entre os limites máximo e mínimo de operação da bancada, ou seja, entre $4,84 \mathrm{~V}$ - que corresponde a $3485 \mathrm{rpm}$ - e $5,00 \mathrm{~V}$ - que corresponde a $3600 \mathrm{rpm}$.

Assim, a planta identificada tem função de transferência tipicamente de primeira ordem, dada por

$$
G(s)=\frac{14.43}{s+1.306}
$$

\subsection{Projeto do controlador PI}

Nesta seção será tratado o controlador PI na forma RST em questão. A função de transferência de malha aberta do sistema identificado no tempo discreto, é dada por (2).

$$
G\left(z^{-1}\right)=\frac{B\left(z^{-1}\right)}{A\left(z^{-1}\right)}
$$

O controlador digital RST é caracterizado pelos polinômios (Landau and Zito, 2007):

$$
\begin{aligned}
R\left(z^{-1}\right) & =r_{0}+r_{1} z^{-1} \\
S\left(z^{-1}\right) & =1-z^{-1} \\
T\left(z^{-1}\right) & =t_{0}+t_{1} z^{-1}
\end{aligned}
$$

Sendo assim, a função de transferência de malha fechada com relação à entrada é dada por:

$$
G_{C L}\left(z^{-1}\right)=\frac{B\left(z^{-1}\right) T\left(z^{-1}\right)}{P_{C L}\left(z^{-1}\right)}
$$

em que:

$$
P_{C L}\left(z^{-1}\right)=A\left(z^{-1}\right) S\left(z^{-1}\right)+B\left(z^{-1}\right) R\left(z^{-1}\right)
$$

O polinômio característico $P_{C L}\left(z^{-1}\right)$ deve ser sintonizado de acordo com as especificações de desempenho desejadas, ou seja, a partir do posicionamento de polos no interior do círculo de raio unitário que atenda às especificações de projeto.

Para o projeto do controlador descrito acima, se faz necessário discretizar a função de transferência em tempo contínuo apresentada na equação (1). Neste projeto considerou-se a aproximação backward com tempo de amostragem de 0,1 $s$, levando à função de transferência discreta em malha aberta dada por:

$$
G\left(z^{-1}\right)=\frac{1,353 z^{-1}}{1-0,8773 z^{-1}}
$$


Assim, a partir da equação (8) pode-se projetar tanto o controlador RST descrito nesta subseção quanto o LQG descrito na seção seguinte.

\subsection{Projeto do controlador $L Q G$}

Em complemento à análise do controlador PI, este trabalho traz o projeto e implementação de um controlador em espaço de estados, no tempo discreto, baseado no controlador linear quadrático gaussiano (LQG) com ação integral.

O controlador LQG é formado pela lei de controle derivada do problema do regulador ótimo quadrático (LQR) em que os estados realimentados são obtidos a partir de um estimador de estados ótimo, o filtro de Kalman. Portanto, o modelo a ser considerado tanto para encontrar o ganho de realimentação de estados - problema LQR -, quanto para achar o ganho de Kalman - problema do estimador ótimo - desempenha papel essencial.

Neste trabalho, considera-se o modelo em tempo discreto, em espaço de estados obtido a partir do modelo autorregressivo:

$$
A\left(z^{-1}\right) y(k)-v(k)=B\left(z^{-1}\right) u(k)+P\left(z^{-1}\right) w(k),
$$

em que

$$
\begin{array}{r}
A\left(z^{-1}\right)=1+a_{1} z^{-1}+\cdots+a_{n} z^{-n} \\
B\left(z^{-1}\right)=b_{0} z^{-1}+b_{1} z^{-2}+\cdots \\
\cdots+b_{m} z^{-m}
\end{array}
$$

são polinômios que descrevem o modelo da planta que se deseja controlar, tal que $n=m+1$. Os polinômios $A\left(z^{-1}\right)$ e $B\left(z^{-1}\right)$ dados pelas equações (10) e (11) correspondem àqueles definidos na equação $2 \mathrm{e}$, portanto, definem a função de transferência da planta $G\left(z^{-1}\right)$. As variáveis $v(k)$ e $w(k)$ são sinais de ruído branco e representam perturbações no sistema.

O termo $P\left(z^{-1}\right)$ é um polinômio cujo posicionamento dos polos é caracterizado como parâmetro de projeto. Este modelo é bastante utilizado nas estratégias de controle preditivo, e para o qual Park et al. (2008) mostram que o algoritmo GPC, quando se considera o polinômio- $T$, pode ser separado em um controlador de horizonte deslizante RHC (Receding Horizon Controller) e um filtro de Kalman em regime permanente. Comumente temse $T\left(z^{-1}\right)$ dado por

$$
\begin{aligned}
P\left(z^{-1}\right) & =\left(1-\alpha z^{-1}\right)^{n} \\
& =1+p_{1} z^{-1}+\cdots+p_{n} z^{-n}
\end{aligned}
$$

Porém, o modelo da equação 9 necessita ser escrito em espaço de estados, cuja realização na forma canônica observável leva a:

$$
\begin{aligned}
\mathbf{x}(k+1) & =\mathbf{A x}(k)+\mathbf{B} u(k)+\mathbf{E} w(k) \\
y(k) & =\mathbf{C x}(k)+w(k)+v(k)
\end{aligned}
$$

sendo

$$
\begin{aligned}
& \mathbf{A}=\left[\begin{array}{ccccc}
-a_{1} & 1 & 0 & \cdots & 0 \\
-a_{2} & 0 & 1 & \cdots & 0 \\
\vdots & \vdots & \vdots & \ddots & \vdots \\
-a_{n-1} & 0 & 0 & \cdots & 1 \\
-a_{n} & 0 & 0 & \cdots & 0
\end{array}\right] ; \quad \mathbf{B}=\left[\begin{array}{c}
b_{1} \\
b_{2} \\
\vdots \\
b_{m}
\end{array}\right] \\
& \mathbf{C}=\left[\begin{array}{llll}
1 & 0 & \cdots & 0
\end{array}\right] ; \mathbf{E}=\left[\begin{array}{c}
p_{1}-a_{1} \\
p_{2}-a_{1} \\
\vdots \\
p_{n}-a_{n}
\end{array}\right]
\end{aligned}
$$

A inclusão do integrador na equação (9) se dá pela inclusão de um estado adicional com este propósito. Isto leva à representação em estado aumentado dada por

$$
\begin{aligned}
{\left[\begin{array}{c}
\mathbf{x}(k+1) \\
\mathbf{x}_{\mathbf{i}}(k+1)
\end{array}\right] } & =\left[\begin{array}{cc}
\mathbf{A} & \mathbf{0} \\
-\mathbf{C} & \mathbf{I}
\end{array}\right]\left[\begin{array}{c}
\mathbf{x}(k) \\
\mathbf{x}_{\mathbf{i}}(k)
\end{array}\right]+ \\
& {\left[\begin{array}{c}
\mathbf{B} \\
\mathbf{0}
\end{array}\right] \mathbf{u}(k)+\left[\begin{array}{c}
\mathbf{0} \\
\mathbf{I}
\end{array}\right] \mathbf{r}(k), }
\end{aligned}
$$

em que

$$
\mathbf{x}_{\mathbf{i}}(k+1)=\mathbf{x}_{\mathbf{i}}(k)+r(k)-\mathbf{C x}(k)
$$

corresponde ao estado adicionado para inclusão da ação integral.

Portanto, a equação algébrica associada ao problema LQR é obtida considerando-se as matrizes aumentadas descritas na equação (15):

$$
\begin{aligned}
\mathbf{S}(\mathbf{k}+\mathbf{1})= & \overline{\mathbf{A}}^{T} \mathbf{S}(\mathbf{k}) \overline{\mathbf{A}}-\overline{\mathbf{A}}^{T} \mathbf{S} \overline{\mathbf{B}} \times \\
& {\left[\overline{\mathbf{B}}^{T} \mathbf{S}(\mathbf{k}) \overline{\mathbf{B}}+\mathbf{R}\right]^{-1} \times } \\
& \overline{\mathbf{B}}^{T} \mathbf{S}(\mathbf{k}) \overline{\mathbf{A}}+\mathbf{Q} .
\end{aligned}
$$

sendo $\overline{\mathbf{A}}=\left[\begin{array}{cc}\mathbf{A} & \mathbf{0} \\ -\mathbf{C} & \mathbf{I}\end{array}\right] \quad$ e $\quad \overline{\mathbf{B}}=\left[\begin{array}{c}\mathbf{B} \\ \mathbf{0}\end{array}\right]$

A soluçã̃o única da equação (16) leva à obtenção do ganho de realimentação de estados

$$
\mathbf{K}_{\mathbf{L}}=\left(\overline{\mathbf{B}}^{T} \mathbf{S} \overline{\mathbf{B}}+\mathbf{R}\right)^{-1} \overline{\mathbf{B}}^{T} \mathbf{S} \overline{\mathbf{A}} .
$$

Por outro lado, a equação algébrica de Riccatti associada ao problema da filtragem de Kalman fica dada por:

$$
\begin{gathered}
\mathbf{P}(\mathbf{k}+\mathbf{1})=\mathbf{A} \mathbf{P}(\mathbf{k}) \mathbf{A}^{T}+\mathbf{E} R_{w} \mathbf{E}^{T}- \\
\left(\mathbf{A P}(\mathbf{k}) \mathbf{C}^{T}+\mathbf{E} R_{w} \mathbf{F}^{T}\right) \times \\
\left(\mathbf{C P}(\mathbf{k}) \mathbf{C}^{T}+R_{v}+\mathbf{F} R_{w} \mathbf{F}^{T}\right)^{-1} \times \\
\left(\mathbf{A P}(\mathbf{k}) \mathbf{C}^{T}+\mathbf{E} R_{w} \mathbf{F}^{T}\right)^{T} .
\end{gathered}
$$

cuja solução leva ao ganho de Kalman:

$$
\begin{aligned}
\mathbf{K}_{\mathbf{F}}=\left(\mathbf{A} \mathbf{P} \mathbf{C}^{T}+\mathbf{E} R_{w} \mathbf{F}^{T}\right) \times \\
\left(\mathbf{C P C} \mathbf{C}^{T}+R_{v}+\mathbf{F} R_{w} \mathbf{F}^{T}\right)^{-1} .
\end{aligned}
$$


A partir da equação (13) percebe-se claramente que o posicionamento do polo $\alpha$ na equação (12) tem efeito na matriz $\mathbf{E}$ e, consequentemente, na resposta do sistema em relação ao sinal de perturbação, ou seja, o polinômio $P\left(z^{-1}\right)$ descrito na equação (12) tem efeito na robustez do controlador. Esse tipo de sintonia foi investigada no âmbito do controle de velocidade de uma máquina de relutância variável (Correia et al., 2016), em que avaliou-se o caso de $P\left(z^{-1}\right)$ assumir polos complexos conjugados. No caso deste trabalho, porém, avalia-se o efeito de sintonia relacionada a um sistema de primeira ordem e, portanto, $\alpha \in \mathbb{R}$ e $n=1$ na equação (12).

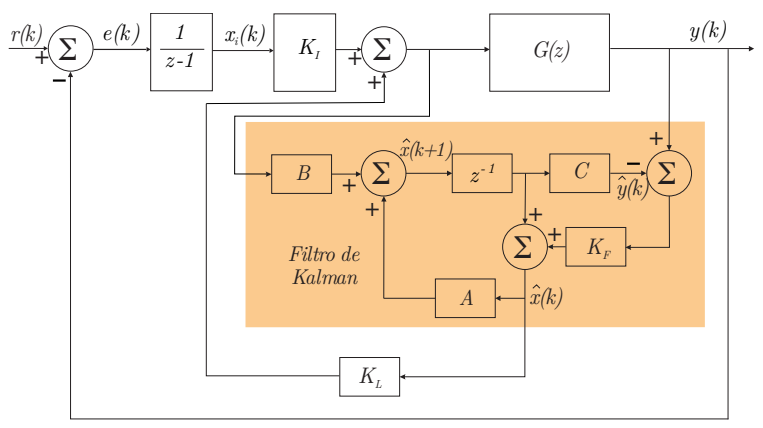

Figura 5: Diagrama de blocos do controlador LQG com ação integral em tempo discreto.

\section{Resultados}

\subsection{Resultados de simulação}

Esta seção apresenta os resultados obtidos em simulação para os controladores considerados nas seções 3.4 e 3.5. Ambos foram sintonizados com desempenho dinâmico semelhante, ou seja, tempo de subida, tempo de acomodação e máximo sobressinal aproximadamente iguais, conforme pode-se verificar na Figura 6. A simulação considera uma perturbação do tipo degrau unitário aplicada em $t=3 \mathrm{~s}$, além da aplicação de ruído com média nula e variância var $=1 \cdot 10^{-6}$ em $t=7 \mathrm{~s}$.

A Tabela 1 apresenta os critérios de desempenho desejados para o projeto dos controladores. O controlador PI na forma RST tem ação integral

Tabela 1: Critérios de desempenho PI RST.

\begin{tabular}{cr}
\hline Critério & Setpoint \\
\hline \hline Tempo de acomodação (s) & 2 \\
Máximo sobressinal (\%) & 1 \\
\hline
\end{tabular}

dada pelo polinômio $S\left(z^{-1}\right)=1-z^{-1}$. Assim, a partir dos valores apresentados na Tabela 1 e considerando o projeto de posicionamento de polos (Landau and Zito, 2007), obtém-se os polinômios
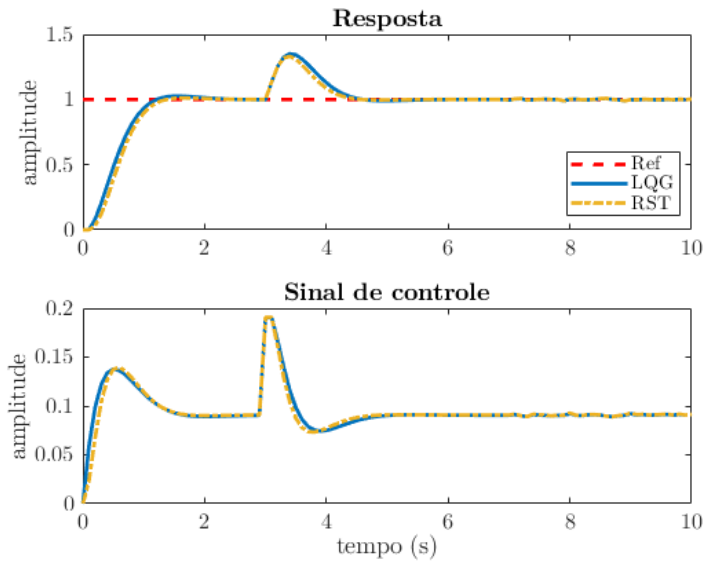

Figura 6: Resposta ao degrau dos controladores.

$$
\begin{aligned}
T\left(z^{-1}\right) \text { e } R\left(z^{-1}\right): & \\
R\left(z^{-1}\right) & =0,2201-0,1765 z^{-1} \\
T\left(z^{-1}\right) & =0,02345+0,02019 z^{-1}
\end{aligned}
$$

O controlador LQG da subseção 3.5 tem as matrizes de ponderação da equação (16) dadas por:

$$
\mathbf{R}=200 \quad \mathbf{Q}=1 .
$$

Vale destacar que tratam-se de valores escalares porque o sistema que se deseja controlar, descrito pela equação (8), possui apenas uma entrada e é de primeira ordem. Essa condição implica ainda que o polinômio de robustez da equação (12) seja igualmente de ordem 1 , sendo dado por

$$
P\left(z^{-1}\right)=1-0.1 z^{-1}
$$

\subsection{Resultados experimentais}

Os resultados em bancada foram obtidos a partir da implementação descrita na seção 3.1 conforme apresentado na Figura 3. Dessa forma, o controlador de torque, ou seja, o controlador da malha externa implementado em computador, atua de forma assíncrona com o controlador das malhas elétricas. Assim, somente a referência de velocidade é passada para atuação dos controladores de corrente e tensão.

No instante de tempo $t \approx 25 \mathrm{~s}$ aplicou-se uma perturbação do tipo degrau unitário, com duração aproximada de 5 segundos, obtida a partir do afundamento de tensão do motor em teste. Para isso, a tensão de alimentação do motor 2 na Figura 4 foi reduzida de $380 \mathrm{~V}$ para $310 \mathrm{~V}$. Entretanto, essa operação é realizada manualmente, razão pela qual os resultados são levemente diferentes quando os controladores são comparados, conforme percebe-se na Figura 7

Desta forma, ambos os controladores são adequados à aplicação em bancada com atuação assíncrona da malha de variáveis mecânicas. Entretanto, os resultados experimentais mostram a 


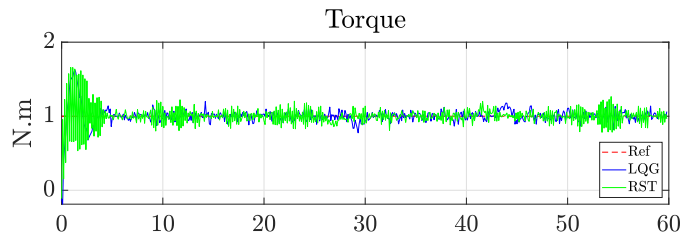

Velocidade

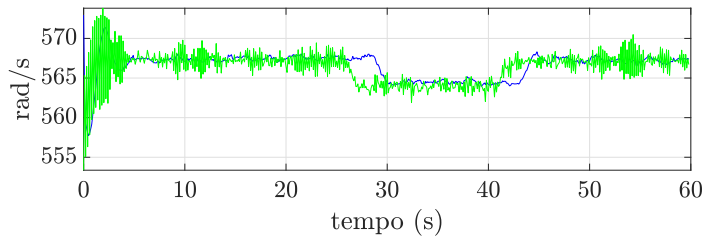

Figura 7: Resultado experimental dos controladores aplicados à planta considerada neste trabalho.

superioridade do controlador LQG em relação ao ruído. O sinal de controle do controlador RST é visivelmente mais ruidoso que o sinal de controle LQG.

\section{Conclusões}

Apresenta-se neste trabalho a implementação de duas estratégias de controle diferentes em acionamento do motores elétricos. O objetivo é controlar o torque, a fim de que se possa emular cargas reais. Nesse contexto, uma bancada de motores elétricos pode ser utilizada para emular cargas para um motor de teste. O objetivo não é comparar as respostas de uma estratégia de controle com outra (PI e LQG) mas simplesmente avaliar a resposta de controladores ao perfil de carga de torque constante, em solução assíncrona em relação aos controladores de velocidade e corrente. Aquele embarcado em microcomputador, este em inversor de frequência para acionamento de motores elétricos. Assim, torna-se possível considerar os algoritmos de controle da malha de torque embarcados em placas de desenvolvimento, em futuras aplicações. Isto é especialmente útil ao se priorizar a implementação de perfis de carga diferentes do torque constante apresentado neste trabalho.

\section{Agradecimentos}

Os autores agradecem o suporte financeiro do CNPq.

\section{Referências}

Agamloh, E. B., won Kim, D. and Jeong, J.Y. (2011). An automated test system for induction motor efficiency measurement to ieee and iec standards, Seventh International Conference EEMODS'11, Energy Efficiency in Motor Drive System - Alexandria, $V A, E U A$.
Arellano-Padilla, J., Asher, G. M. and Sumner, M. (2006). Control of an ac dynamometer for dynamic emulation of mechanical loads with stiff and flexible shafts, IEEE Transactions on Industrial Electronics 53(4): 1250-1260.

Correia, W. B., de Carvalho Almeida, R. N., da Silva, W. A., 3, B. C. T., Pinto, V. P. and dos Reis, L. L. N. (2016). The t-polynomial approach for lqg control applied to a switched reluctance motor (srm), PRZEGLD ELEKTROTECHNICZNY 92: 161-167.

de Oliveira, C. M. R., de Aguiar, M. L., Guazzelli, P. R. U., de Castro, A. G., d. Santos, S. T. C. A. and Monteiro, J. R. B. A. (2018). Analysis of the dynamic emulation problem for validation of position control algorithms in machine drives, 2018 13th IEEE International Conference on Industry Applications (INDUSCON), pp. 1266-1273.

Gan, C., Todd, R. and Apsley, J. M. (2015). Drive system dynamics compensator for a mechanical system emulator, IEEE Transactions on Industrial Electronics 62(1): 70-78.

Gynther, L., Mustonen, S. and Saarivirta, E. (2016). Policies and Measures for Promoting Efficient Electric Motors in Industry, World Energy Council (WEC).

Landau, I. D. and Zito, G. (2007). Digital control systems: design, identification and implementation, Springer Science \& Business Media.

Ouhrouche, M., Errouissi, R., Trzynadlowski, A. M., Tehrani, K. A. and Benzaioua, A. (2016). A novel predictive direct torque controller for induction motor drives, IEEE Transactions on Industrial Electronics 63(8): 5221-5230.

Park, J. J., Han, S. and Kwon, W. H. (2008). Finite memory generalized predictive controls for discrete-time state space models, International Conference on Control, Automation and Systems - Seoul, Korea.

Quintino, A., Abrantes, N., Ferreira, F. J. T. E. and de Almeida, A. T. (2011). Four-quadrant electric motor test bench with a fully programmable load profile, Seventh International Conference EEMODS'11, Energy Efficiency in Motor Drive System - Alexandria, $V A, E U A$.

Waide, P. and Brunner, C. U. (2011). EnergyEfficiency Policy Opportunities for Electric Motor-Driven Systems, International Energy Agency (IEA). 